\title{
Chaotic particle heating due to an obliquely propagating wave in a magnetized plasma
}

\author{
T. M. Corrêa da Silva, ${ }^{*}$ R. Pakter, and F. B. Rizzato \\ Instituto de Física, Universidade Federal do Rio Grande do Sul, Porto Alegre, Rio Grande do Sul, Brazil \\ M. C. de Sousa, I. L. Caldas, and F. M. Steffens \\ Instituto de Física, Universidade de São Paulo, São Paulo, São Paulo, Brazil \\ (Received 20 March 2013; revised manuscript received 29 May 2013; published 3 July 2013)
}

\begin{abstract}
We study the dynamics of a relativistic charged particle in the presence of a uniform magnetic field and a stationary electrostatic wave that propagates at an arbitrary angle. The wave is considered as a series of periodic pulses which allows us to derive an exact map for the system. In particular, we investigate the heating process of an initially low-energy particle. It is found that abrupt changes in the maximum energy attained by the particle may occur as the angle between the wave propagation and the magnetic field varies. To determine what is the mechanism behind this phenomenon a reduced Hamiltonian that retains the important dynamical features is obtained. Using both Poincaré plots and perturbation theory, we identify that a separatrix reconnection is the key mechanism for the abrupt change in particle response.
\end{abstract}

DOI: 10.1103/PhysRevE.88.013101

PACS number(s): 52.20.Dq, 05.45.-a, 45.50.Dd, 52.50.Qt

\section{INTRODUCTION}

Wave-particle interaction is a well-known method for particle acceleration and particle heating. There are several applications in which the wave-particle interaction arises, like in particle accelerators [1-3], free-electron devices [2,4], and heating and current generation in fusion devices $[5,6]$, among others. While in some cases the phenomena can be described and understood by modeling the wave as a single monochromatic mode [3,5-7], more generally the particle may interact with a spectrum of waves [8-10]. In particular, when the spectrum is broad and the group velocity is large, the wave can be well approximated by a series of periodic pulses [11]. This allows for the integration of the resulting dynamical equations over one wave period and the derivation of a map to describe the particle evolution. The map facilitates the analysis not only because it largely speeds up the computation of the system evolution, but also because it may yield a series of exact analytical results to work with [11-18].

In the realm of area preserving maps to describe waveparticle interaction one of the most studied systems is the standard map in one degree of freedom [12]. Despite its simplicity, the standard map can successfully show how appropriate resonant conditions give rise to efficient particle acceleration and chaotic heating. Along the years many generalizations of the standard map have been proposed to include important effects, such as the relativistic mass variation of the particles as they gain energies comparable to their rest mass $[11,15]$. However, despite the fact that the wave-particle interaction in a vast number of systems takes place in the presence of an external magnetic field [2,3,5-10], there was no map to describe such cases. The difficulty in deriving such maps is that while in the absence of a background magnetic field the canonical variables appear separately in different parts of the Hamiltonian - the momentum in the kinetic part only and the coordinate in the interacting part only-the presence of the magnetic field mixes the variables in the different parts

*thalestr@gmail.com of the Hamiltonian, making the integration along one wave period more cumbersome. Only recently it has been shown that by using a set of appropriate canonical transformations one can derive an exact relativistic map that also includes a background magnetic field perpendicular to the direction of the wave propagation [19]. The map led to analytical estimates on the necessary conditions to generate efficient regular acceleration. The possibility of chaos suppression using a secondary copropagating wave has also been investigated in this system [20]. Still, in many situations of interest in both laboratory and space plasmas, the wave propagates at an arbitrary angle with respect to the background magnetic field [21-23].

In this paper, we generalize the model considered in Ref. [19] by allowing the wave to propagate at an arbitrary angle with respect to the magnetic field. In this case the wave drives the particle dynamics in both the transverse and the parallel direction with respect to the magnetic field, increasing the number of degrees of freedom in the system. Using appropriate canonical transformations, we are able to derive an exact map for the system. We focus on the study of how initially low-energetic particles can gain energy from the wave. It is found that the heating process of such particles is highly dependent on the wave propagation angle and that abrupt changes in the maximum energy attained by the particle occur as the angle is varied. In order to investigate the mechanism responsible for such a phenomenon we expand the system Hamiltonian in terms of its Fourier modes to determine which modes are the most relevant. It is found that the simplest normal form corresponds to a two degrees of freedom, nonintegrable Hamiltonian. Using both Poincaré plot techniques and perturbation theory we find that the abrupt change in the system behavior is caused by the reconnection between the separatrix that governs the low-energy particle dynamics and that of a resonant island generated by a coupling between parallel and transverse particle motion. Comparisons between the full system and the reduced normal form indicate that, despite the high dimensionality of the full system, Arnold diffusion plays very little role in the dynamics. 
The paper is organized as follows: in Sec. II we present the model and derive the associated map; in Sec. III we investigate with the aid of the map the dynamics of low-energy particles and find that their energy gain is highly dependent on the wave propagation angle; in Sec. IV we obtain and analyze a simplified Hamiltonian in order to understand the mechanisms that lead to the results found in Sec. III; in Sec. V we use perturbation theory to further simplify the reduced Hamiltonian and then obtain analytical estimates from the model; in Sec. VI, we present our conclusions.

\section{MODEL AND DERIVATION OF THE MAP}

Let us consider a particle of mass $m$, charge $q$, and momentum $\mathbf{p}$ moving under the combined action of a uniform magnetic field $\mathbf{B}=B_{0} \hat{\mathbf{z}}$ and a stationary electrostatic wave of wave vector $\mathbf{k}$, period $T$, and amplitude $\varepsilon$. The wave propagates at an arbitrary angle $\varphi$ with respect to the magnetic field. Working with a set of dimensionless quantities, $\mathbf{p} / m c \rightarrow \mathbf{p},\left(q B_{0} / m c\right)(x, z) \rightarrow(x, z),\left(q B_{0} / m\right)(t, T) \rightarrow$ $(t, T)$, and $\left(1 / m c^{2}\right)\left(q B_{0} / m\right) \varepsilon \rightarrow \varepsilon$, the dimensionless Hamiltonian $H \rightarrow H / m c^{2}$ governing the motion of the particle can be written as

$$
H=\sqrt{1+x^{2}+p_{x}^{2}+p_{z}^{2}}+\varepsilon \cos \left(k_{x} x+k_{z} z\right) \sum_{n=-\infty}^{\infty} \delta(t-n T)
$$

where $c$ is the speed of light in vacuo and without loss of generality we consider that the wave vector lies along the $x-z$ plane, i.e., $\mathbf{k}=k_{x} \hat{\mathbf{x}}+k_{z} \hat{\mathbf{z}}=k \sin \varphi \hat{\mathbf{x}}+k \cos \varphi \hat{\mathbf{z}}$, and that the canonical momentum along the $y$ direction is zero. Note that despite the fact that $p_{y}$ is zero, $d y / d t$ is nonvanishing along the dynamics and the motion in the transverse $x-y$ plane is not one dimensional.

As stated earlier, we focus on pulsed waves represented by a periodic collection of $\delta$ functions. In this case, we are able to integrate the particle dynamics along one wave period $T$. To this purpose, we first notice that because the kicking term depends on $x$ and $z$, the respective momenta, $p_{x}$ and $p_{z}$, undergo abrupt changes across the kicks. On the other hand, between consecutive kicks the Hamiltonian is time independent, $p_{z}$ is conserved, and the dynamics is completely integrable. This fact becomes apparent if one makes use of action-angle variables $I$ and $\theta$ given by $p_{x}=\sqrt{2 I} \cos \theta$ and $x=\sqrt{2 I} \sin \theta$. The Hamiltonian written in terms of these coordinates becomes

$$
\begin{aligned}
H= & \sqrt{1+2 I+p_{z}^{2}} \\
& +\varepsilon \cos \left(k_{x} \sqrt{2 I} \sin \theta+k_{z} z\right) \sum_{n=-\infty}^{\infty} \delta(t-n T),
\end{aligned}
$$

and it is clear that not only $p_{z}$ is conserved between consecutive kicks, but also the action $I$. With these in mind, we adopt the following procedure to integrate the dynamics along one wave period via successively applying convenient coordinate transformations.

(i) Just before the $n$th kick, we have $I_{n}, \theta_{n}, p_{z_{n}}$, and $z_{n}$.

(ii) Action and angle are transformed to the original Cartesian variables via $\left(x_{n}, p_{x_{n}}\right)=\left(\sqrt{2 I_{n}} \sin \theta_{n}, \sqrt{2 I_{n}} \cos \theta_{n}\right)$. (iii) Across the kick, we compute the changes in the Cartesian coordinates: $\Delta x=\Delta z=0$ and $\Delta p_{x / z}=$ $\varepsilon k_{x / z} \sin \left(k_{x} x+k_{z} z\right)$.

(iv) Immediately after the kick we write $p_{x_{n}}^{+}=p_{x_{n}}+$ $\Delta p_{x}, p_{z_{n}}^{+}=p_{z_{n}}+\Delta p_{z}, x_{n}^{+}=x_{n}+\Delta x$, and $z_{n}^{+}=z_{n}+\Delta z$ and change back to action-angle variables via $I_{n}^{+}=\left(p_{x_{n}}^{+2}+\right.$ $\left.x_{n}^{+2}\right) / 2$ and $\theta_{n}^{+}=\arctan \left(x_{n}^{+} / p_{x_{n}}^{+}\right)$.

(v) The last step is to propagate the preceding state to the instant right before the $n+1$ kick via $I_{n+1}=I_{n}^{+}$, $\theta_{n+1}=\theta_{n}^{+}+T / \sqrt{1+2 I_{n+1}+p_{z_{n+1}}^{2}}, p_{z_{n+1}}=p_{z_{n}}^{+}$, and $z_{n+1}=$ $z_{n}^{+}+p_{z_{n+1}} T / \sqrt{1+2 I_{n+1}+p_{z_{n+1}}^{2}}$.

We can express the final map in terms of the Cartesian coordinates, but we choose to use the action-angle variables because the action is conserved in the absence of the wave. The result can be cast in the form of a map relating the dynamical variables just before consecutive kicks $n$ and $n+1$,

$$
\begin{aligned}
I_{n+1}= & I_{n} \sin ^{2} \theta_{n}+\frac{1}{2}\left[\sqrt{2 I_{n}} \cos \theta_{n}+\varepsilon k_{x} f\left(I_{n}, \theta_{n}, z_{n}\right)\right]^{2}, \\
\theta_{n+1}= & \arctan \left[\frac{\sqrt{2 I_{n}} \sin \theta_{n}}{\sqrt{2 I_{n}} \cos \theta_{n}+\varepsilon k_{x} f\left(I_{n}, \theta_{n}, z_{n}\right)}\right] \\
& +g\left(I_{n+1}, p_{z_{n+1}}\right), \\
p_{z_{n+1}}= & p_{z_{n}}+\varepsilon k_{z} f\left(I_{n}, \theta_{n}, z_{n}\right), \\
z_{n+1}= & z_{n}+g\left(I_{n+1}, p_{z_{n+1}}\right) p_{z_{n+1}},
\end{aligned}
$$

where

$$
\begin{gathered}
f\left(I_{n}, \theta_{n}, z_{n}\right)=\sin \left(k_{x} \sqrt{2 I_{n}} \sin \theta_{n}+k_{z} z_{n}\right), \\
g\left(I_{n}, p_{z_{n}}\right)=\frac{T}{\sqrt{1+2 I_{n}+p_{z_{n}}^{2}}} .
\end{gathered}
$$

Note that it is possible to put the map in a fully explicit form by substituting the expressions for $I_{n+1}$ and $p_{z_{n+1}}$, Eqs. (3) and (5), in Eqs. (4) and (6). Moreover, a careful inspection shows that the map Jacobian has unitary norm, which guarantees the symplectic nature of the theory.

\section{LOW-ENERGY PARTICLE DYNAMICS}

We are now in a position to explore the properties of the system. In particular, we want to investigate the dynamics of initially low-energetic particles to determine if they can gain substantial energy from the wave interaction. To this end, for a given set of parameters we iterate the map (3)-(6) for particles with vanishing small initial $I$ and $p_{z}$ and compute the particle energy $E_{n}=\sqrt{1+2 I_{n}+p_{z_{n}}^{2}}$ in each wave cycle. We perform this for a large number of cycles $n=10^{5}$ and store the maximum energy $E_{\max }$ attained by the particle along its trajectory. From now on, we focus on resonant cases where the wave period is the same as the cyclotron period, $T=2 \pi$, in the normalized units [24].

In Fig. 1(a) we present the results for $E_{\max }$ as a function of the angle $\varphi$, keeping all other parameters fixed. For small angles, the particles seem to very poorly interact with the wave because their maximum energy remains close to the initial one. Nevertheless, at a certain critical value $\varphi_{c} \approx 0.38 \pi$ there is a sudden jump in the energy attained by the particle. Above the critical value, $E_{\max }$ tends to decrease with increasing $\varphi$, 
(a)

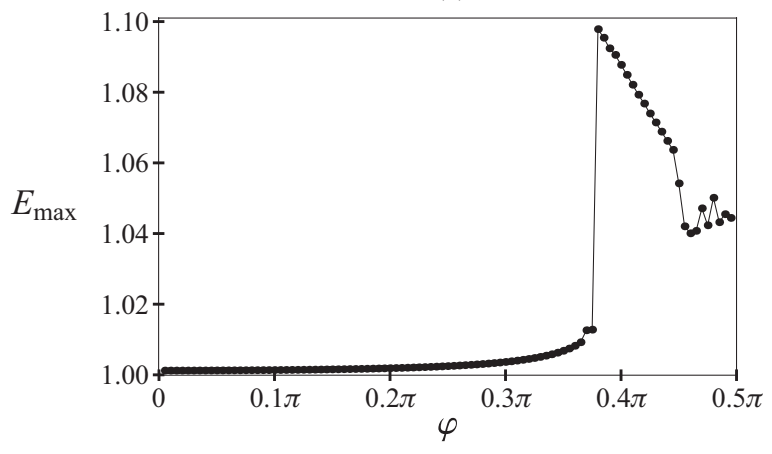

(b)

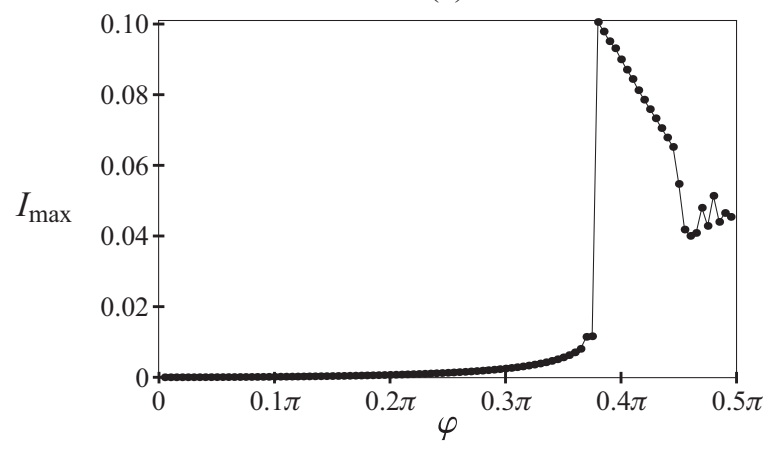

(c)

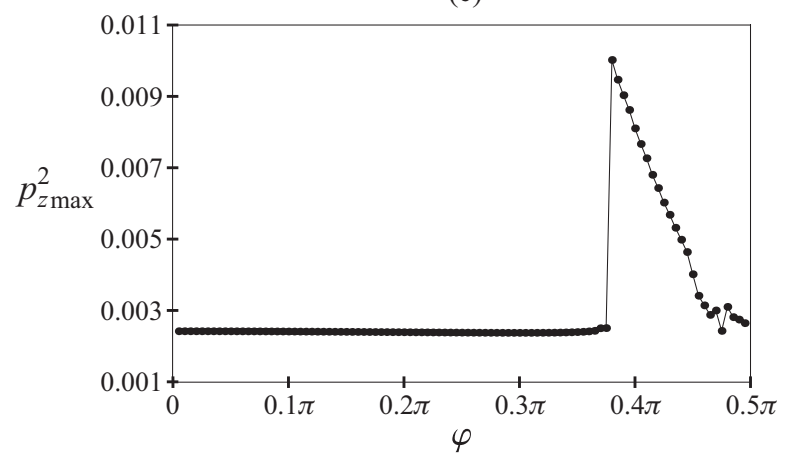

FIG. 1. Results from the simulation of the map (3)-(6) for the maximum energy $E_{\max }(\mathrm{a})$, maximum action $I_{\max }(\mathrm{b})$, and maximum parallel momentum $p_{z \max }(\mathrm{c})$ as a function of the angle between the magnetic field and the wave. The highest values occur at a critical angle $\varphi_{c} \approx 0.38 \pi$, where an abrupt change in the system response occurs. The parameters used are $\varepsilon=0.025$ and $k=1$. The initial condition is $I_{0}=10^{-6}, \theta_{0}=0, p_{z_{0}}^{2}=0$, and $z_{0}=0.75 \pi / k_{z}$.

creating therefore a maximum at the critical value. In other words, properly adjusting the angle between the wave vector and the magnetic field may lead to a large gain in the particle energy. Despite variations in the value of the critical angle, the same scenario is observed for other system parameters. Our goal is then to determine what is the precise mechanism that leads to the onset of particle energization at $\varphi_{c}$. In Figs. 1(b) and 1 (c) we show the maximum values for the action $I_{\max }$ and the parallel momentum $p_{z \max }$ for the same parameters as in Fig. 1(a). It is seen that energy, action, and parallel momentum have a similar response. In particular, they are all peaked at $\varphi_{c} \approx 0.38 \pi$. This suggests that there is a sizable interaction between the parallel and transverse particle motion in this situation and serves as a hint for the possible mechanism for particle energization.

\section{REDUCED HAMILTONIAN}

As stated earlier, it is clear from Eq. (2) that when the wave term is absent the Hamiltonian does not depend on the coordinates $\theta, z$, and $t$, and the system is integrable. Therefore, in order to investigate the properties of the full system we start by expanding the wave contribution in Hamiltonian Eq. (2) in terms of Fourier components of the coordinates $[13,25]$. This leads to

$$
\begin{aligned}
H=\sqrt{1+2 I+p_{z}^{2}} & \\
& +\frac{\varepsilon}{2 \pi} \sum_{l, n} J_{l}\left(k_{x} \sqrt{2 I}\right) \cos \left(l \theta+n t+k_{z} z\right),
\end{aligned}
$$

where the sum in the integers $l$ and $n$ goes from $-\infty$ to $\infty$ and $J_{l}$ are the Bessel functions of the first kind. Among the infinite Fourier components present in the above Hamiltonian, it is expected that most of them just cause small perturbations in the particle's trajectory and are of minor importance. Only some, with particular values of $l$ and $n$, strongly affect the particle by resonantly coupling to its dynamics. These terms should be mainly responsible for the particle energization and need to be identified.

Let us first consider the parallel motion. It is convenient to introduce the following canonical transformation,

$$
\begin{aligned}
\bar{\theta} & =\theta, \quad I=\bar{I}+\frac{l \bar{p}_{z}}{k_{z}}, \\
\bar{z} & =z+\frac{l \theta}{k_{z}}+\frac{n t}{k_{z}}, \quad p_{z}=\bar{p}_{z}, \\
\bar{H} & =H+\frac{n \bar{p}_{z}}{k_{z}},
\end{aligned}
$$

where the bar indicates the new variables. Selecting only a single term with given $n$ and $l$ and neglecting the rest of the infinite sum in (9), the reduced Hamiltonian in the new variables is

$$
\begin{aligned}
\bar{H}_{l, n}= & \sqrt{1+2 \bar{I}+\frac{2 l \bar{p}_{z}}{k_{z}}+\bar{p}_{z}^{2}}+\frac{n \bar{p}_{z}}{k_{z}} \\
& +\frac{\varepsilon}{2 \pi} J_{l}\left[k_{x} \sqrt{2 \bar{I}+\frac{2 l \bar{p}_{z}}{k_{z}}}\right] \cos \left(k_{z} \bar{z}\right) .
\end{aligned}
$$

Because it does not depend on $\bar{\theta}$ and $t$, both $\bar{I}$ and $\bar{H}_{l, n}$ are constants of motion. Examples of phase-space plots obtained from $\bar{H}_{l, n}\left(\bar{z}, \bar{p}_{z}\right)$ are shown in Fig. 2. While in panel (a) for $l=$ $n=0$ we see a large pendulumlike resonance with the elliptic fixed point located at $\bar{p}_{z}=0, \bar{z}=\pi / k_{z}$, panel (b) for $l=0$ and $n=1$ shows almost straight lines corresponding to $\bar{p}_{z} \approx$ const. We have tested other values of $l$ and $n$ and they all lead to a phase space similar to the one depicted in Fig. 2(b). We can then conclude that the parallel motion is mostly governed by the $l=n=0$ term. Note, however, that a continuous change in the angle $\varphi$ (i.e., in the ratio $k_{z} / k_{x}$ ) leads only to a continuous change in the shape of the pendulumlike resonance of Fig. 2(a). 
(a)

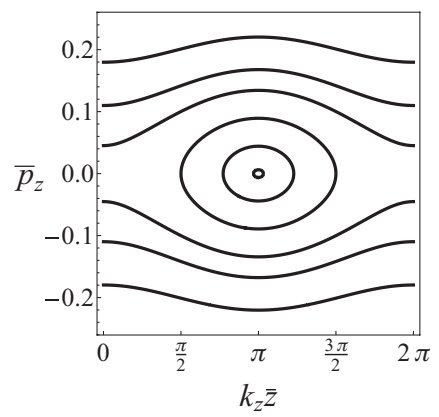

(b)

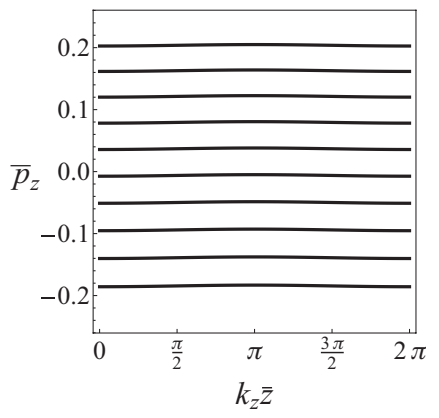

FIG. 2. Phase-space plots of $\bar{H}_{l, n}\left(\bar{z}, \bar{p}_{z}\right)$ given by Eq. (11) for $l=n=0$ in (a) and $l=0$ and $n=1$ in (b). The parameters are $\bar{I}=10^{-6}, k=1, \varepsilon=0.025$, and $\varphi=0.4 \pi$.

Therefore, this term alone cannot explain the abrupt change in the particle energy gain at $\varphi_{c}$.

In order to investigate the role of the transversal motion, it is now convenient to consider the following canonical transformation from the original variables,

$$
\begin{array}{r}
\hat{\theta}=\theta+\frac{k_{z} z}{l}+\frac{n t}{l}, \quad I=\hat{I}, \\
\hat{z}=z, \quad p_{z}=\hat{p}_{z}+\frac{k_{z} \hat{I}}{l}, \\
\hat{H}=H+\frac{n \hat{I}}{l},
\end{array}
$$

where the hat indicates the new variables. Again, by selecting only a single term with given $n$ and $l$ a reduced, integrable Hamiltonian $\hat{H}_{l, n}(\hat{\theta}, \hat{I})$ that does not depend on either $\hat{z}$ or $t$ is obtained. Analyzing the phase spaces obtained from $\hat{H}_{l, n}(\hat{\theta}, \hat{I})$ one readily finds that the $(\hat{I}, \hat{\theta})$ dynamics is mostly affected by the Fourier terms with $l=-n= \pm 1$. Analogous to $\bar{H}_{l, n}\left(\bar{z}, \bar{p}_{z}\right)$ in Eq. (11), the reduced Hamiltonian $\hat{H}_{l, n}(\hat{I}, \hat{\theta})$ also varies continuously with the ratio $k_{z} / k_{x}$. Therefore, each $l=-n=$ \pm 1 term alone cannot explain the existence of a critical $\varphi$ and we may need to add together the contribution coming from the $l=n=0$ term previously discussed. It is worth noting that a quick inspection of Eq. (9) reveals that, whereas the $l=n=0$ term is indeed associated with pure parallel motion, the $l=-n= \pm 1$ terms clearly come from a coupling between parallel and transverse motions.

Taking into consideration the three resonant terms in Eq. (9), we can obtain a resonant Hamiltonian that we expect to incorporate all the important dynamical features of the system, in particular the mechanism that explains the abrupt energy gain at a critical $\varphi$. Because the only dependence of the resonant Hamiltonian on $\theta$ and $t$ comes from the $l=-n= \pm 1$ and appears in the form $\theta-t$, it is convenient to perform an additional canonical transformation

$$
\theta-t \rightarrow \theta, \quad H-I \rightarrow H .
$$

The resonant Hamiltonian then reads

$$
\begin{aligned}
H_{\mathrm{res}}= & \sqrt{1+2 I+p_{z}^{2}}-I+\frac{\varepsilon}{2 \pi}\left\{J_{0}\left(k_{x} \sqrt{2 I}\right) \cos \left(k_{z} z\right)\right. \\
& \left.+2 J_{1}\left(k_{x} \sqrt{2 I}\right)\left[\cos \left(k_{z} z+\theta\right)-\cos \left(k_{z} z-\theta\right)\right]\right\} .
\end{aligned}
$$

(a)

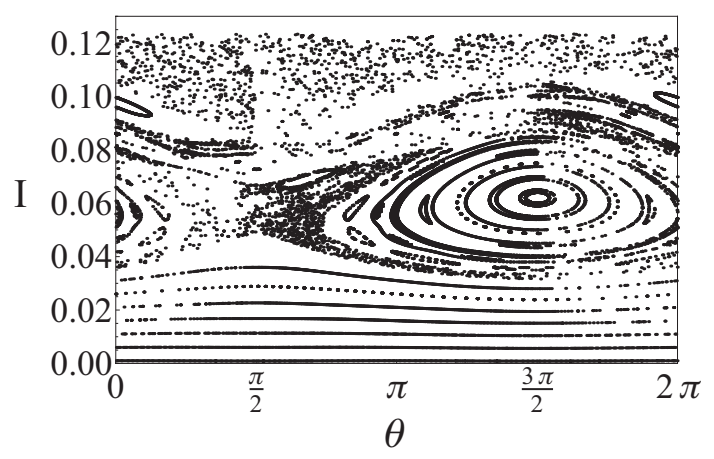

(b)

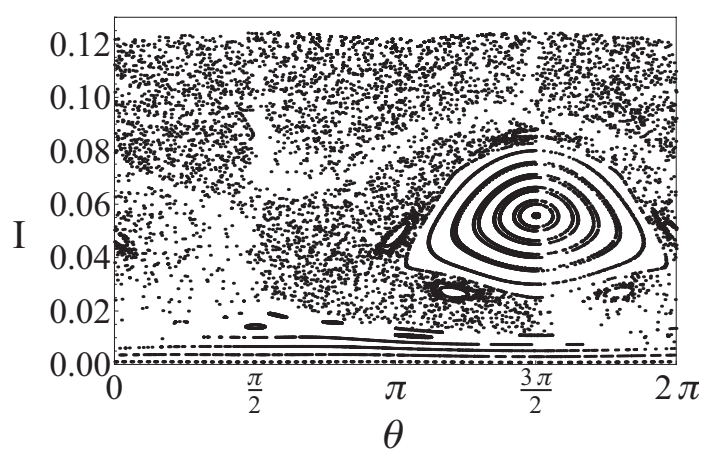

(c)

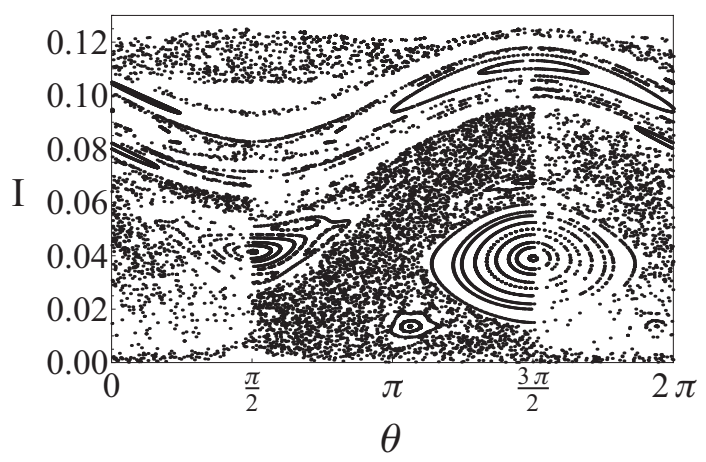

FIG. 3. Poincaré plots of the resonant Hamiltonian in Eq. (14) for $\varphi=0.1 \pi$ in (a), $\varphi=0.25 \pi$ in (b), and $\varphi=0.38 \pi$ in (c). The remainder parameters are $k=1, \varepsilon=0.025$, and $z_{0}=0.75 \pi / k_{z}$.

In principle, this Hamiltonian is the simplest normal form that incorporates the desired dynamical features. Yet, because it depends on both parallel variables $\left(z, p_{z}\right)$ and transversal variables $(\theta, I)$, it may be chaotic. We therefore need to apply nonlinear dynamical techniques to analyze it, in particular Poincaré plots $[13,26]$. We choose to plot the $(\theta, I)$ phase space each time $p_{z}=0$ with $d p_{z} / d t>0$. The results are shown in Fig. 3 for the same parameters used in Fig. 1. In panel (a), with $\varphi=0.1 \pi$, we see that despite the existence of a large resonance centered at $I \approx 0.06$, the low-energy particles are not affected by it and remain in the low action region of the phase space. Increasing the angle to $\varphi=0.25 \pi$, panel (b), we note that the resonance moves a little towards lower action values and increases in size, creating stronger chaotic activity 
in its surroundings. However, the low-energy particles are still not affected and do not gain significant amounts of energy. By further increasing the angle to $\varphi=0.38 \pi$, panel (c), we observe that the chaotic layer spreads all the way to $I \rightarrow 0$ and starts to heat the initially low-energetic particles. Note also that the chaotic layer has an upper limit of $I \approx 0.1$, which agrees very well with the $I_{\max }$ found in Fig. 1(b).

It is important to mention that because the resonant Hamiltonian (14) has two degrees of freedom, the chaotic layer seen in Fig. 3(c) is really bounded; a limiting curve [27] at $I \approx 0.1$ acts as a barrier that completely limits the particle energy gain. On the other hand, the full system (2) is higher-dimensional and Arnold diffusion could play a role $[13,22]$. Nevertheless, we have compared the maximum values of action obtained from the reduced $H_{\text {res }}$ with those found by iterating the full map for a very large number of wave periods and the results agree well. In particular, while the chaotic diffusion through the phase space of Fig. 3(c) occurs along $10^{1}-10^{2}$ wave periods, even after $10^{6}$ map iteration the particle is still bounded to move in the region limited to $I \approx 0.1$. This indicates that Arnold diffusion, if present, is very weak with exponentially small diffusion coefficients.

\section{PERTURBATION THEORY AND ESTIMATE OF $\varphi_{c}$}

Since the simplified resonant Hamiltonian (14) describes the important features of the wave-particle interaction, in particular the occurrence of the abrupt particle energy gain as the angle between wave and magnetic field is varied, we would like to use it to estimate $\varphi_{c}$. For that, we use perturbation theory.

Assuming that $I,\left|p_{z}\right| \ll 1$ when the wave amplitude is small, we expand $H_{\text {res }}$ in power series of these quantities. Retaining the leading-order terms we get

$$
\begin{aligned}
H_{\mathrm{res}}= & -\frac{I^{2}}{2}+\frac{\varepsilon k_{x} \sqrt{2 I}}{4 \pi}\left[\cos \left(k_{z} z+\theta\right)-\cos \left(k_{z} z-\theta\right)\right] \\
& +\frac{p_{z}^{2}}{2}+\frac{\varepsilon}{2 \pi} \cos \left(k_{z} z\right) .
\end{aligned}
$$

Let us for a moment neglect the coupling between parallel and transverse degrees of freedom [second term on the right-hand side of Eq. (15)]. In this case the Hamiltonian describes a pendulumlike dynamics for $\left(z, p_{z}\right)$; in fact, the one depicted in Fig. 2(a). Suppose that the system is close enough to the elliptic fixed point of the pendulum such that it can be approximated by an harmonic oscillator. The solution for $z(t)$ then becomes

$$
z(t)=\frac{\pi}{k_{z}}-a \cos \left(\omega_{z} t\right)
$$

where $a=\pi / k_{z}-z_{0}$ and $\omega_{z}=\sqrt{\varepsilon k_{z}^{2} / 2 \pi}$.

Substituting Eq. (16) back into the Hamiltonian (15), we obtain that the transverse dynamics is dictated by

$$
H_{\mathrm{res}}=-\frac{I^{2}}{2}-\frac{\varepsilon k_{x} \sqrt{2 I}}{2 \pi} J_{1}\left(a k_{z}\right)\left[\sin \left(\theta+\omega_{z} t\right)+\sin \left(\theta-\omega_{z} t\right)\right],
$$

where use has been made of $a k_{z} \ll 1$, which is consistent with the fact that the system is close to the elliptic fixed point of Fig. 2(a). This Hamiltonian is not yet integrable because it

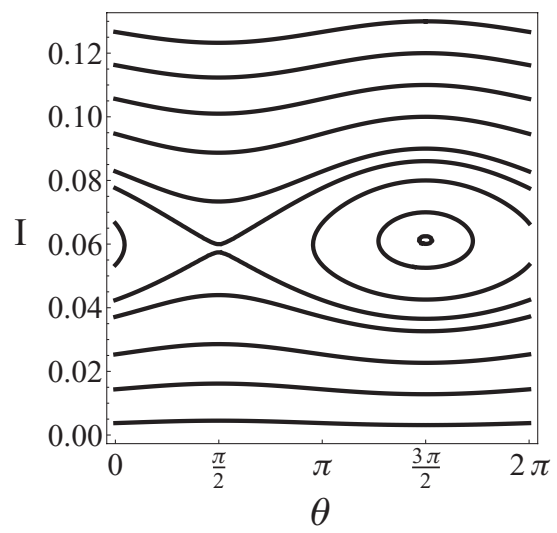

FIG. 4. Phase space obtained from the simplified resonant Hamiltonian in Eq. (18). The parameters correspond to those in Fig. 3(a), namely $\varphi=0.1 \pi, k=1, \varepsilon=0.025$, and $k_{z} a=0.25 \pi$.

depends on both $\theta+\omega_{z} t$ and $\theta-\omega_{z} t$. However, an analysis analogous to that used in Sec. IV, reveals that the important, resonant term is the former one. Neglecting the nonresonant term, the Hamiltonian can finally be written as

$$
H_{\mathrm{res}}=\omega_{z} I-\frac{I^{2}}{2}-\frac{\varepsilon k_{x} \sqrt{2 I}}{2 \pi} J_{1}\left(a k_{z}\right) \sin \theta
$$

where an additional canonical transformation $\theta+\omega_{z} t \rightarrow \theta$, $H_{\text {res }}+\omega_{z} I \rightarrow H_{\text {res }}$ has been performed. Figure 4 presents the phase space obtained from this Hamiltonian for the same parameters as in Fig. 3(a). Despite the expected nonexistence of high-order resonances and chaos, it is clear that Eq. (18) gives a good description of the dynamics. In particular, it shows the absence of energy gain for particles close to $I=0$ and the large resonance in the vicinity of $I \approx 0.06$. We recall that this resonance is generated by the coupling between parallel and transverse particle motion.

Let us concentrate on the dynamics of initially lowenergetic particles. Substituting $I=0$ in Eq. (18) leads to $H_{\text {res }}=0$. Therefore, the trajectory of such particles corresponds to the $H_{\text {res }}=0$ level curves of (18). In Fig. 5 we plot these curves as $\varphi$ is increased. For $\varphi=0.370 \pi$, panel (a), we see that $H_{\text {res }}=0$ has two branches, which indicates that $H_{\text {res }}$ is a nonmonotonic function of the action $I$. The lower branch (dashed curve) is the one that describes the trajectory of initially low-energy particles. It corresponds to a nonpendular separatrix [14] that divides the trajectories with limited $\theta$ motion (in the $0<\theta<\pi$ range) from those with unbounded $\theta$ motion. The upper branch (solid curve) is just a spurious solution of $H_{\text {res }}=0$ that is not directly related to the dynamics of the low-energy particles for these parameter values. However, it is important to point out that the pendular resonant island generated by the parallel-transverse coupling (the one centered at $I \approx 0.06$ in Fig. 4) lies in between these two branches of $H_{\text {res }}=0$. As we increase the angle to $\varphi=0.379 \pi$, panel (b), we can still see the two branches, but they are getting closer to each other. When $\varphi=0.381 \pi$, panel (c), the branches intersect each other at $\theta=\pi / 2$ forming a single orbit that can now drive the low-energetic particles to higher energy states. As a matter of fact, this orbit is formed by the reconnection between the separatrixes of the low-energy particles and of the resonant island generated by 
(a)

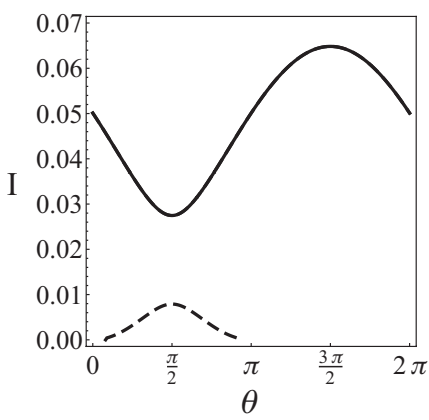

(b)

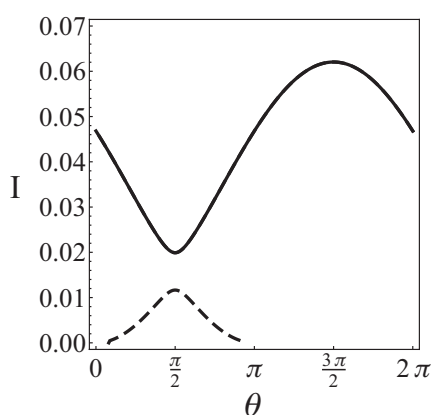

(c)

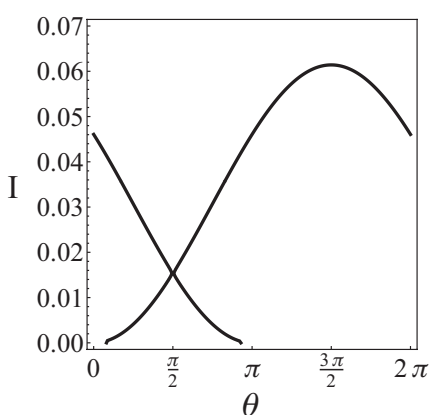

(d)

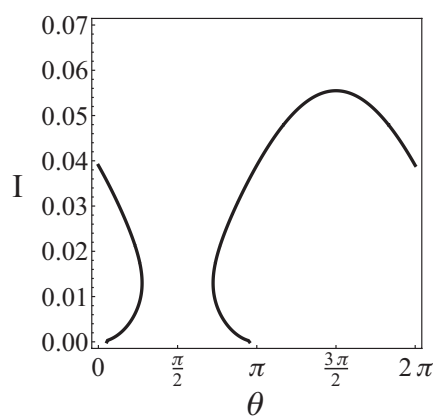

FIG. 5. Level curves $H_{\text {res }}=0$ of the simplified resonant Hamiltonian in Eq. (18) for $\varphi=0.370 \pi$ in (a), $\varphi=0.379 \pi$ in (b), $\varphi=0.381 \pi$ in (c), and $\varphi=0.4 \pi$ in (d). The remainder parameters are $k=1, \varepsilon=0.025$, and $k_{z} a=0.25 \pi$.

the parallel-transverse coupling. This corresponds to $\varphi_{c}$. For $\varphi>\varphi_{c}$, such as in panel (d), $H_{\text {res }}=0$ corresponds to a single curve that still accelerates the particles initially at $I=0$. However, the maximum $I$ of the curve (located at $\theta=3 \pi / 2$ ) decreases with $\varphi$, such that the maximum energy is lower than that with $\varphi_{c}$. This is in agreement with the results in Fig. 1 that show that the maximum $I_{\max }$ occurs exactly at critical angle.

Based on this scenario and on Fig. 5(c) we notice that we can find $\varphi_{c}$ by searching for the angle at which $H_{\text {res }}=0$ presents a single solution at $\theta=\pi / 2$. This condition leads to

$$
4 \sqrt{2 \pi} k \cos ^{3} \varphi_{c}-27 \sqrt{\varepsilon} J_{1}^{2}\left(a k_{z}\right) \sin ^{2} \varphi_{c}=0 .
$$

To verify this estimate we have run the full system (3)-(6) for different values of the wave number $k$ and have searched for the critical angle at which the abrupt energy gain occurs. The comparison between the simulation results and the theoretical prediction of Eq. (19) is presented in Fig. 6, showing a good agreement.

As a final remark let us just point out the importance of properly taking into account the relativistic effects in the model. Its relevance becomes apparent by inspecting the resonant Hamiltonian Eq. (18). Neglecting the relativistic mass correction corresponds to dropping the nonlinear $I^{2}$ term in $H_{\text {res }}$. In this case, the level curves of $H_{\text {res }}$ would not present the resonance generated by the parallel-transverse coupling (the large resonance in the vicinity of $I \approx 0.06$ in Fig. 4 ) and

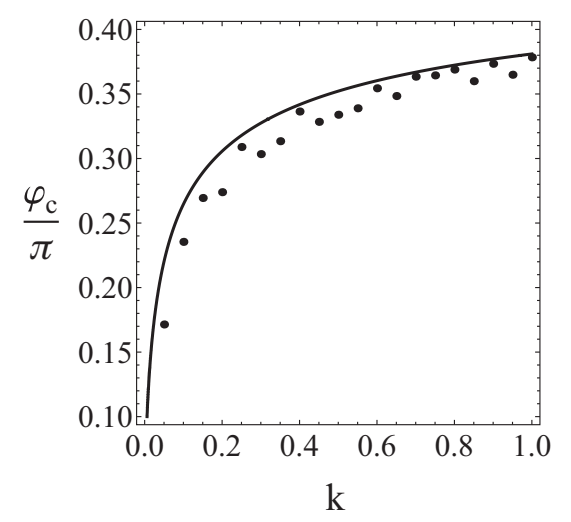

FIG. 6. Comparison between the critical angle $\varphi_{c}$ obtained by simulating the full system (3)-(6) (symbols) and the theoretical prediction of Eq. (19). the separatrix reconnection scenario described in Fig. 5 would be absent. Therefore, if the relativistic effect were neglected, the whole process of particle heating discussed here would be overlooked.

\section{CONCLUSIONS}

We studied the dynamics of a relativistic charged particle in the presence of a uniform magnetic field and a stationary electrostatic wave. The wave is allowed to propagate at an arbitrary angle with respect to the magnetic field and is considered to be a train of periodic pulses. An exact map was derived for the wave-particle interaction and shows that the heating process of low-energetic particles is highly dependent on the wave propagation angle. In particular, abrupt changes in the maximum energy attained by the particle occur as the angle is varied. In order to investigate the mechanism responsible for such a phenomenon we expanded the system Hamiltonian in terms of its infinite Fourier modes and determined which modes are the most relevant. It was found that the simplest normal form corresponds to a two degrees of freedom, nonintegrable Hamiltonian. Using both Poincaré plot techniques and perturbation theory it was possible to determine that the abrupt change in the system behavior is caused by a separatrix reconnection. At a certain angle $\varphi_{c}$ the separatrix of a higher-energy resonant island generated by the coupling between the parallel and transverse particle motion collides with the separatrix that governs the low-energy dynamics. The low-energy particles then start to be chaotically driven to higher-energy states. In fact, it was found that $\varphi_{c}$ is not only a threshold for the chaotic heating, but also corresponds to the wave propagating angle at which the heating is most efficient. An analytical estimate of $\varphi_{c}$ was presented and tested against results from the full map. Finally, comparisons between the results of the full system and the reduced normal form indicate that despite the former being a high-dimensional system Arnold diffusion plays very little role in the dynamics.

\section{ACKNOWLEDGMENTS}

This work was supported by CNPq, FAPERGS, and FAPESP, Brazil, and by the US-AFOSR under Grant No. FA9550-12-1-0438. 
[1] T. Tajima and J. M. Dawson, Phys. Rev. Lett. 43, 267 (1979).

[2] P. K. Shukla, N. N. Rao, M. Y. Yu, and N. L. Tsintsadze, Phys. Rep. 138, 1 (1986).

[3] R. Pakter, R. S. Schneider, and F. B. Rizzato, Phys. Rev. E 49, 1594 (1994).

[4] J. T. Mendonça, Theory of Photon Acceleration (IOP Publishing, Bristol, 2001).

[5] C. F. F. Karney, Phys. Fluids 21, 1584 (1978).

[6] N. J. Fisch, Rev. Mod. Phys. 59, 175 (1987).

[7] J.-H. Kim and H.-W. Lee, Phys. Rev. E 54, 3461 (1996).

[8] D. Benisti, A. K. Ram, and A. Bers, Phys. Plasmas 5, 3224 (1998).

[9] L. M. Tozawa and L. F. Ziebell, Phys. Rev. E 66, 056409 (2002).

[10] C. B. Wang, C. S. Wu, and P. H. Yoon, Phys. Rev. Lett. 96, 125001 (2006).

[11] A. A. Chernikov, T. Tel, G. Vattay, and G. M. Zaslavsky, Phys. Rev. A 40, 4072 (1989).

[12] B. V. Chirikov, Phys. Rep. 52, 265 (1979).

[13] A. J. Lichtenberg and M. A. Lieberman, Regular and Chaotic Dynamics (Springer, New York, 1992).

[14] G. Corso and F. B. Rizzato, Phys. Rev. E 58, 8013 (1998).

[15] Y. Nomura, Y. H. Ichikawa, and W. Horton, Phys. Rev. A 45, 1103 (1992).
[16] W. Simeoni, Jr., F. B. Rizzato, and R. Pakter, Phys. Plasmas 13, 063104 (2006).

[17] S. S. Abdullaev, Phys. Rev. E 76, 026216 (2007).

[18] J. D. Szezech, Jr., I. L. Caldas, S. R. Lopes, R. L. Viana, and P. J. Morrison, Chaos 19, 043108 (2009).

[19] M. C. de Sousa, F. M. Steffens, R. Pakter, and F. B. Rizzato, Phys. Rev. E 82, 026402 (2010).

[20] M. C. de Sousa, I. L. Caldas, F. B. Rizzato, R. Pakter, and F. M. Steffens, Phys. Rev. E 86, 016217 (2012).

[21] C. R. Menyuk, A. T. Drobot, K. Papadopoulos, and H. Karimabadi, Phys. Rev. Lett. 58, 2071 (1987).

[22] H. Karimabadi and V. Angelopoulos, Phys. Rev. Lett. 62, 2342 (1989).

[23] R. Kamendje, S. V. Kasilov, W. Kernbichler, and M. F. Heyn, Phys. Plasmas 10, 75 (2003).

[24] J.-M. Rax, L. Laurent, and D. Moreau, Europhys. Lett. 15, 497 (1991); J. T. Mendonça, Phys. Scr. T 63, 136 (1996).

[25] C. S. Kueny and P. J. Morrison, Phys. Plasmas 2, 4149 (1995).

[26] R. Pakter, G. Corso, T. S. Caetano, D. Dillenburg, and F. B. Rizzato, Phys. Plasmas 1, 4099 (1994); R. Pakter, S. R. Lopes, and R. L. Viana, Physics D 110, 277 (1997); F. B. Rizzato and R. Pakter, Phys. Rev. Lett. 89, 184102 (2002).

[27] M. Frichembruder, R. Pakter, G. Gerhardt, and F. B. Rizzato, Phys. Rev. E 62, 7861 (2000). 